\title{
Internet of Things (IoTs) and Marketing: Conceptual Issues, Applications, and a Survey: An Abstract
}

\author{
Syed Tariq Anwar
}

\begin{abstract}
The purpose of this paper is to discuss and evaluate the area of IoTs in marketing and its related sectors. The IoTs have grown multifold and will have a big impact on marketing and consumers in the USA and global markets. The paper reviews the IoTs-related literature and its interdisciplinary and multidisciplinary perspectives and related investigations. We specifically used over 90 conceptual and empirical studies which were published between 2009 and 2017. For interdisciplinary and multidisciplinary research, we benchmarked studies such as Cheng et al. (2009, 2014), Fulmer and Ployhart (2014), Picone et al. (2014), and Siedlok and Hibbert (2014). Our common denominator and topics regarding finding relevant studies on IoTs was based on smart devices, controllers, sensors, actuators, Webbased areas, and technological advances. In addition to EBSCO and Business Source Complete, we used Web-based searches and publishers' indexes (see Wiley, Elsevier/ Science Direct, Springer, SAGE, Taylor \& Francis, Palgrave, Routledge, etc.) which complemented the literature. These criteria helped us regarding retrieving interdisciplinary and multidisciplinary studies, surveys, and related investigations.

In the areas of IoTs and their connected markets and connected consumers, we continue to witness emerging business models, IT-related platforms, and digital ecosystems. As of 2018, the IoTs and their multifaceted areas and technologies stand out in the connected world of consumers and industrial sectors alike. The areas of IoTs clearly enjoy their distinct growth and wide-scale applications in domestic and global markets.

In conclusion, the areas of IoTs play an important role in marketing and other sectors of the business world in domestic and global markets. In this paper, we presented a review-based discussion of the IoTs areas and their research domains and applications. Within today's changing markets and consumer demand, Internetrelated technologies are bound to increase their sales and growth. In marketing, the areas of IoTs clearly enjoy a good growth potential and wide scale of applications. The paper also provides future research directions and other avenues of this debate.
\end{abstract}

S. T. Anwar $(\bowtie)$

West Texas A\&M University, Canyon, TX, USA

e-mail: sanwar@mail.wtamu.edu 